\title{
Deep phenotyping, including quantitative ciliary beating parameters, and extensive genotyping in primary ciliary dyskinesia
}

\author{
Sylvain Blanchon (D) , ${ }^{1,2}$ Marie Legendre, ${ }^{3}$ Mathieu Bottier, ${ }^{1}$ Aline Tamalet, ${ }^{4}$ \\ Guy Montantin, ${ }^{3}$ Nathalie Collot, ${ }^{3}$ Catherine Faucon, ${ }^{5}$ Florence Dastot, ${ }^{3}$ Bruno Copin, ${ }^{3}$ \\ Annick Clement, ${ }^{3,4}$ Marcel Filoche, ${ }^{3}$ André Coste, ${ }^{6}$ Serge Amselem, ${ }^{3}$ Estelle Escudier, ${ }^{3}$ \\ Jean-Francois Papon, ${ }^{1,7}$ Bruno Louis ${ }^{1}$
}

For numbered affiliations see end of article.

\section{Correspondence to}

Dr Sylvain Blanchon, Universite Paris-Est, Faculte de Médecine, INSERM (Institut National pour la Santé et la Recherche Médicale) UMR_S955, Equipe 13, CNRS (Centre National pour la Recherche Scientifique), Creteil ERL7000, France; sylvain.blanchon@chuv.ch

Received 11 July 2019 Revised 20 September 2019 Accepted 13 October 2019 Published Online First 26 November 2019

\section{Check for updates}

(C) Author(s) (or their employer(s)) 2020. No commercial re-use. See rights and permissions. Published by BMJ.

To cite: Blanchon $\mathrm{S}$,

Legendre M, Bottier M, et al. $J$ Med Genet

2020;57:237-244

\begin{abstract}
Background Primary ciliary dyskinesia (PCD) is a rare genetic disorder resulting in abnormal ciliary motility/ structure, extremely heterogeneous at genetic and ultrastructural levels. We aimed, in light of extensive genotyping, to identify specific and quantitative ciliary beating anomalies, according to the ultrastructural phenotype.
\end{abstract}

Methods We prospectively included 75 patients with PCD exhibiting the main five ultrastructural phenotypes ( $n=15 /$ group), screened all corresponding PCD genes and measured quantitative beating parameters by highspeed video-microscopy (HSV).

Results Sixty-eight (91\%) patients carried biallelic mutations. Combined outer/inner dynein arms (ODA/IDA) defect induces total ciliary immotility, regardless of the gene involved. ODA defect induces a residual beating with dramatically low ciliary beat frequency (CBF) related to increased recovery stroke and pause durations, especially in case of DNA/1 mutations. IDA defect with microtubular disorganisation induces a low percentage of beating cilia with decreased beating angle and, in case of CCDC39 mutations, a relatively conserved mean CBF with a high maximal CBF. Central complex defect induces nearly normal beating parameters, regardless of the gene involved, and a gyrating motion in a minority of ciliated edges, especially in case of RSPH1 mutations. $P C D$ with normal ultrastructure exhibits heterogeneous HSV values, but mostly an increased CBF with an extremely high maximal CBF.

Conclusion Quantitative HSV analysis in PCD objectives beating anomalies associated with specific ciliary ultrastructures and genotypes. It represents a promising approach to guide the molecular analyses towards the best candidate gene(s) to be analysed or to assess the pathogenicity of the numerous sequence variants identified by next-generation-sequencing.

\section{INTRODUCTION}

Primary ciliary dyskinesia (PCD) is a rare congenital disorder (1:10000-40 000 births) related to abnormal structure and/or motility of motile cilia. ${ }^{1-4}$ The cilia dysfunction leads to recurrent upper/lower airway infections secondary to impaired mucociliary clearance, associated with laterality defect in approximately half of the patients, defining the
Kartagener syndrome..$^{5-7}$ The diagnosis, usually suspected in case of suggestive clinical picture and low nasal nitric oxide ( $\mathrm{NNO}$ ), is challenged by the heterogeneity of the disease and the limitations of the available diagnostic tools. ${ }^{8-10}$

A wide diversity of ciliary ultrastructural defects characterise PCD, mainly affecting the dynein arms or less frequently the central complex of the axoneme. It reflects an important genetic heterogeneity with more than 40 genes involved, ${ }^{8}$ each related to a specific ultrastructural phenotype. ${ }^{4}$ Specific ciliary beating anomalies related to specific ultrastructural defects were first described in the 1980s, ${ }^{11} 12$ later refined through high-speed video-microscopy (HSV). ${ }^{13}$ Additionally, one study correlated beating anomalies and genotypes, but with few patients for most of the genes. ${ }^{14}$ Recently, Chioccioli reported subtle beating differences between patients with DNAH11 and HYDIN variants. ${ }^{15}$ Furthermore, studies focused on specific genotypes described the related ultrastructure and/ or ciliary beating. ${ }^{16-19}$ Therefore, despite international recommendations underlying the importance in genotype/ultrastructure/beating correlations in $\mathrm{PCD},{ }^{8} 9$ no exhaustive description or correlation study is available. Moreover, in all these studies but one, ${ }^{15}$ the ciliary beating analysis was based on a subjective description of the beating pattern and/or on the ciliary beat frequency (CBF).

Applied to cilia since $1984,{ }^{20} \mathrm{HSV}$ dramatically improved beating analysis, allowing multiple slow-motion-replays and providing video collections suitable for expert advice or research. ${ }^{8} 1321$ In 2017, the European Respiratory Society underlined the importance of HSV in PCD diagnosis, ${ }^{8}$ recently sustained by his excellent sensitivity/ specificity (100\%/96\%, respectively). ${ }^{22}$ However, the lack of standardisation in beating analysis, the variability in beat pattern and frequency even in healthy control, and the risk of observer bias in a subjective beating analysis, question the accuracy of such analyses and stimulate the interest of objective and standardised analyses. ${ }^{892324}$ Consequently, the American Thoracic Society recently recommended to not use subjective beat pattern analysis for PCD diagnosis. ${ }^{9}$ In a pilot study, our team developed quantitative HSV beating parameters to improve 
PCD diagnosis, ${ }^{25}$ that would be valuable to reduce the observer bias and to develop an objective standardised method with high accuracy and high decisiveness.

We aimed to describe the ciliary beating in a large PCD cohort, using quantitative/objective ciliary beating parameters, according to the ultrastructural phenotype and the genotype.

\section{METHODS}

\section{Patients}

Patients suspected of PCD are routinely referred to the French national centre of rare respiratory diseases (https://respifil.fr/) for investigations, including $\mathrm{nNO}$, beating analysis by HSV, ultrastructural analysis by transmission electron microscopy (TEM), genetic analysis and if necessary ciliary analyses after cell culture.

We selected patients with confirmed or highly likely PCD diagnosis, available HSV and ultrastructural analysis evidencing one of the following main five ultrastructural phenotypes, encompassing $>90 \%$ of PCD cases: ${ }^{8}$ combined outer/inner dynein arms defect (2 DA group), outer dynein arm defect (ODA group), inner dynein arm defect with microtubular disorganisation (IDA/MTD group), central complex defect (CC group) and normal ultrastructure (nEM group) (see examples in online supplementary figure S1). As recommended, ${ }^{8}$ confirmed PCD diagnosis required a hallmark ultrastructural defect (ie, 2DA defect, ODA defect, IDA defect with MTD) or biallelic causal mutations in a known PCD gene. PCD diagnosis was considered as highly likely in case of Kartagener syndrome or in case of typical clinical picture associated with CC defect in $>20 \%$ of cilia confirmed on two independent samples, since situs inversus has never been reported and the defect never affects all cilia in patients with CC defect. ${ }^{8}$ 26-29

In order to constitute five groups corresponding to the main five ultrastructural phenotypes, we prospectively and consecutively included 15 patients for each phenotype (75 patients). In the 2DA, ODA and IDA/MTD groups, we selected patients evidencing dynein defect in $100 \%$ of cilia, in order to get a more homogeneous group. In the CC group, PCD diagnosis was confirmed by the identification of causal mutations $(n=13 / 15)$ or highly likely in affected siblings with the same typical clinical picture (chronic bronchitis/rhinosinusitis with diffuse bronchiectasis) and ultrastructural defect $(n=2 / 15)$. The $n E M$ group included patients with $>90 \%$ of cilia with normal ultrastructure and either biallelic DNAH11 mutations $(n=13)$ or Kartagener syndrome $(n=2)$. Overall, we included 71 patients with confirmed PCD and 4 with highly likely PCD (CC group $(n=2)$, nEM group $(n=2))$.

All patients/parents gave their written informed consent. The Ile-de-France ethics committee approved the study (CPP07729).

\section{Ciliary analyses}

The ciliated epithelium samples were obtained from the inferior turbinate or the bronchus, as previously described, ${ }^{28}$ in nonsmokers patients free from acute airway infection for at least 6 weeks.

The ciliary ultrastructure was analysed by TEM on biopsy immediately immersed in glutaraldehyde. Results are expressed as the percentage of abnormal cilia among the analysed cilia ( $\geq 50$ per patient) and as the percentage of each ultrastructural abnormality among the abnormal cilia. Dynein arms were considered to be absent when missing in $\geq 5$ over nine peripheral microtubules.

The ciliary beating was analysed by HSV on brushing immediately immersed in B1-BSA medium (CCD, Paris, France).
We analysed samples blinded from clinical/TEM/genetic results, within 3 hours, during a maximum of $20 \mathrm{~min}$, at stable air-conditioned room temperature, close to the physiological temperature of the nostril, considering no international consensus standardised HSV methods notably the temperature. ${ }^{8}$ We excluded isolated ciliated cells. Using an oil immersion $\times 100$ objective, we captured the videos with a digital high-speed camera (A741,PixeLink, Ottawa, Canada), at 358 frames per second with a definition of $256 \times 192$ pixels (1800 frames, $5 \mathrm{~s}$ per video). For each patient, we recorded 10 video captures of 10 independent ciliated edges viewed from the side and additionally from the top in case of circular gyrating motion. The 10 video allowed ciliary beating analyses from 10 independent ciliated edges ( 1 analysis per video/edge), using an in-house software, including:

- The percentage of beating cilia. Each of the 10 video/edges were scored 0 (all immotile cilia), 0.1 ( $<50 \%$ of beating cilia), 0.5 ( $\sim 50 \%$ of beating cilia) or 1 ( $>50 \%$ of beating cilia). For each patient, the percentage of beating cilia was defined as the sum of the scores divided by 10 .

- The CBF, measured by kymography and fast Fourier transform. For each patient, we subsequently inferred the mean and maximal CBF.

- The qualitative evaluation, referring to the beat patterns described by Chilvers (ie, normal beating, virtually immotile, stiff beating with reduce amplitude and circular gyrating motion). ${ }^{13}$ For each video/edges, including those captured from the top, we considered the main beat pattern. For each patient, we calculated the percentage of video/edges with each beat pattern.

- The quantitative beating parameters as previously described. ${ }^{25}$ It required individualising 10 beating cilia followed during a complete beating cycle (one cilium per video/edges). It allowed measuring 12 objective parameters: $\mathrm{CBF}$, power stroke duration, recovery stroke duration, pause duration after power stroke, pause duration after recovery stroke, total pause duration (sum of the two previous parameters), beating angle, cilia length, distance travelled by the cilium tip per beat, area swept by the cilium per beat, distance travelled by the cilium tip per second and area swept by the cilium per second. Furthermore, the distance travelled by the cilium tip per second was weighted by the percentage of beating cilia.

- According to the thresholds proposed by Papon, ${ }^{25}$ we calculated the proportion of beating cilia with 'normal motion', that we defined as the association of $\mathrm{CBF} \geq 7.1 \mathrm{~Hz}$, beating angle $\geq 57^{\circ}$ and distance travelled by the cilium tip per second $\geq 43.2 \mu \mathrm{m}$.

Online supplementary table S2 displays ciliary beating values in a disease control group previously published. ${ }^{25}$

\section{Molecular analyses}

All the coding regions and intronic boundaries of all known PCD genes related to each ultrastructural phenotype were analysed from genomic DNA, ${ }^{4}$ by Sanger sequencing (primers available on request) or, since 2014 , by next-generation sequencing (NGS) for the large ones ( $\geq 30$ exons) (ARMC4, CCDC103, CCDC114, CCDC151, CCDC39, CCDC40, CCDC65/DRC2, CCNO, CFAP300/C11orf70, CFAP298/C21orf59, DNAAF1/ LRRC5, DNAAF2/KTU, DNAAF3, DNAAF5/HEATR2, DNAH5, DNAH9, DNAH11, DNAI1, DNAI2, DNAJB13, DNAL1, DRC1/ CCDC164, DYX1C1/DNAAF4, GAS2L2, GAS8/DRC4, HYDIN, LRRC56, LRRC6, MCIDAS, NME8/TXNDC3, PIH1D3, RPGR, 
Table 1 Patients' characteristics

\begin{tabular}{|c|c|c|c|c|c|c|}
\hline & $2 \mathrm{DA}$ & ODA & IDA/MTD & CC & nEM & Total \\
\hline & $n=15$ & $n=15$ & $n=15$ & $n=15$ & $n=15$ & $n=75$ \\
\hline Male/Female & $8 / 7$ & $9 / 6$ & $10 / 5$ & 9/6 & $7 / 8$ & $43 / 32$ \\
\hline Age (years) & $14(0-44)$ & $30(4-62)$ & $15(0-50)$ & $29(7-53)$ & $27(1-55)$ & $25(0-62)$ \\
\hline \multicolumn{7}{|l|}{ Clinical features } \\
\hline Laterality defect & $7(47 \%)$ & $6(40 \%)$ & $4(27 \%)$ & $0(0 \%)$ & $11(73 \%)$ & $28(37 \%)$ \\
\hline Neonatal respiratory distress & $8(53 \%)$ & $3(20 \%)$ & $11(73 \%)$ & $5(33 \%)$ & $5(33 \%)$ & $32(43 \%)$ \\
\hline Chronic bronchitis and rhinosinusitis & $15(100 \%)$ & $15(100 \%)$ & $15(100 \%)$ & $15(100 \%)$ & $15(100 \%)$ & $75(100 \%)$ \\
\hline Bronchiectasis & $12(80 \%)$ & $11(73 \%)$ & $9(60 \%)$ & $14(93 \%)$ & $11(73 \%)$ & $57(76 \%)$ \\
\hline Adult male infertility* & $3 / 3$ & $3 / 6$ & $3 / 6$ & $3 / 7$ & $1 / 5$ & $13 / 27$ \\
\hline Available nasal NO & $9(60 \%)$ & $13(87 \%)$ & $10(67 \%)$ & $11(73 \%)$ & $10(67 \%)$ & $50(67 \%)$ \\
\hline Nasal NO (nL/min) & $11(1-93)$ & $10(0-60)$ & $13(0-60)$ & $17(6-194)$ & $22(7-121)$ & $12(0-194)$ \\
\hline Abnormal cilia by TEM (\%) & \multicolumn{3}{|c|}{100 (as inclusion criteria) } & $35(20-58)$ & $4(0-9)$ & $100(0-100)$ \\
\hline Patients with identified causal mutations $†$ & $15 / 15(100 \%)$ & $14 / 14(100 \%)$ & $13 / 13(100 \%)$ & $13 / 15(87 \%)$ & $13 / 15(87 \%)$ & $68 / 72(94 \%)$ \\
\hline \multirow[t]{8}{*}{ Genes (n) } & DNAAF1 (3) & DNAl1 (8) & $\operatorname{CCDC39}(10)$ & RSPH1 (6) & DNAH11 (13) & \\
\hline & ZMYND10 (3) & DNAH5 (6) & $\operatorname{CCDC40}(3)$ & RSPH9 (4) & & \\
\hline & $\operatorname{LRRC6}(2)$ & & & DNAJB13 (2) & & \\
\hline & PIH1D3 (2) & & & HYDIN (1) & & \\
\hline & $\operatorname{CCDC114(2)}$ & & & & & \\
\hline & DNAAF2 (1) & & & & & \\
\hline & DNAAF3 (1) & & & & & \\
\hline & DYX1C1 (1) & & & & & \\
\hline
\end{tabular}

Data are expressed as $\mathrm{n}(\%)$ or median (range).

*Among males 18 years and older. Additionally, four women showed hypofertility in 2DA (n=1), ODA ( $n=1)$ and nEM ( $n=2)$ groups.

†Among patients with available molecular analysis.

CC, central complex defect; 2 DA, combined outer/innerdyne in arms defect; IDA/MTD, inner dynein arm defect with microtubular disorganisation; nEM, normal ultrastructure; NO,

nitric oxide; ODA, outer dynein arm defect; TEM, transmission electron microscopy.

RSPH1, RSPH3, RSPH4A, RSPH9, SPAG1, STK36, TTC25, ZMYND10). The NGS approach was based on DNA mechanical fragmentation followed by targeted capture (SeqCap EZ Library SR protocol, Roche) and library paired-end sequencing (MiSeq, Illumina) with a resulting depth above $50 \times$. Data were analysed through an in-house double pipeline based on Bowtie and Bwa aligners. These analyses were performed in all patients except three without available DNA. Phenotype-genotype correlations were only considered for the genes implicated in at least four patients.

\section{Statistical analysis}

Data were analysed per patient (ie, one measure is available for each patient) considering percentage of beating cilia, mean CBF, maximal CBF, qualitative evaluation, mean of each quantitative beating parameter and proportion of beating cilia with 'normal motion'. Additionally, CBF and quantitative beating parameters were analysed per cilia after pooling all cilia from the same ultrastructural phenotype. CBF and quantitative parameters only concerned beating cilia. Comparisons were performed with Statistica V.7.1 (Statsoft, France), using Kruskal-Wallis test. $\mathrm{P}<0.05$ were considered significant.

\section{RESULTS}

Table 1 summarises clinical, nNO, TEM and genetic findings. According to the ACMG classification, ${ }^{30}$ among the 68 patients carrying biallelic mutations, 43 had a genotype that probably drives the absence of protein or a truncated non-functional protein (ie, homozygous or compound heterozygous for nonsense, frameshift or severe splice defect). Additionally, 25 patients had a genotype consistent with a possible production of a full-length protein with or without an amino-acid change (ie, missense or mild splice defect on one or both allele(s)). Among those 25 patients carrying at least one mild mutation, the involved genes were the following: in the 2DA group: DNAAF3 $\mathrm{n}=1$, ZMYND10 $\mathrm{n}=2$; in the ODA group: DNAI $1 \mathrm{n}=5$, DNAH5 $\mathrm{n}=1$; in the IDA/MTD group: CCDC40 $\mathrm{n}=1$; in the CC group: RSPH1 $\mathrm{n}=6$, RSPH9 $\mathrm{n}=1$ and in the nEM group: DNAH11 $\mathrm{n}=8$. Online supplementary table $\mathrm{S} 3$ details the genotype of all the patients.

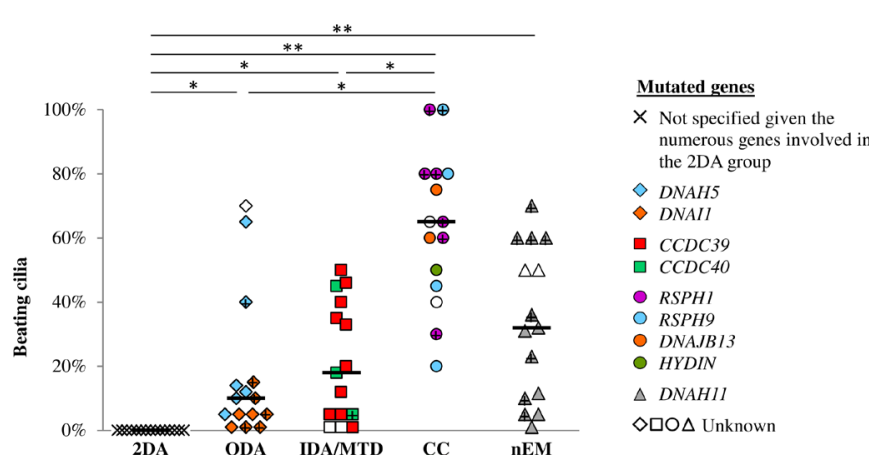

Figure 1 Percentage of beating cilia. Black bars feature medians. + represents patient with potential production of a full-length protein. ${ }^{*} P<0.05,{ }^{* *} p<0.001$. Values in disease controls are displayed in the online supplementary table S2. CC group, central complex defect; IDA/MTD group, inner dynein arm defect with microtubular disorganisation; nEM group, normal ultrastructure; ODA group, outer dynein arm defect; 2 DA group, combined outer/inner dynein arms defect. 

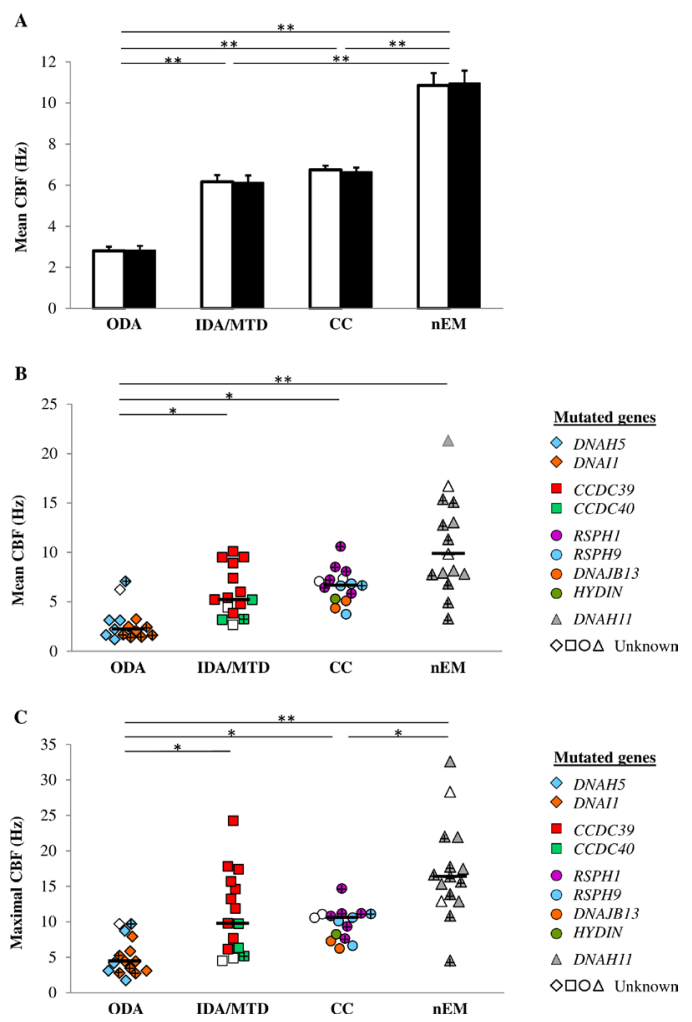

Figure 2 CBF of beating cilia. The 2DA group is not represented since all cilia are immotile. (A) Mean CBF, presented per cilia, as mean and SE, obtained by kymography (white box) and fast Fourier transform (black box). (B) Mean CBF, presented per patient. (C) Maximal CBF per patient. Black bars feature medians. + represents patient with potential production of a full-length protein. ${ }^{*} P<0.05,{ }^{*} p<0.001$. Values in disease controls are displayed in the online supplementary table S2. CBF, ciliary beat frequency; CC group, central complex defect; IDA/MTD group, inner dynein arm defect with microtubular disorganisation; nEM group, normal ultrastructure; ODA group, outer dynein arm defect; 2 DA group, combined outer/inner dynein arms defect.

\section{Ciliary beating and ultrastructural phenotype}

The percentage of beating cilia is displayed in figure 1. Apart patients from the 2DA group, who showed 100\% of immotile cilia, all patients had a residual beating allowing further HSV analyses. Noteworthy, patients from the CC group had at least $20 \%$ of beating cilia, including two with $100 \%$ of beating cilia.

Results of CBF were similar regardless the methods of measurement (ie, fast Fourier transform or kymography) (figure 2A). All CBF comparisons showed significant differences except between the IDA/MTD and CC groups (figure 2A). Furthermore, the patients of the $n E M$ group evidenced a wide range of mean $\mathrm{CBF}$, from 3.3 to $21.3 \mathrm{~Hz}$ (figure 2B). Figure 2C features the maximal CBF per patient, lower in the ODA group and higher in the nEM group, as compared with other groups.

The qualitative evaluation of ciliary beating is summarised in table 2, and the statistical analyses are detailed in online supplementary table 4 . The pattern 'normal beating' was more frequent in the CC or nEM group; the pattern 'virtually immotile' was more frequent in the 2DA group; and the pattern 'stiff beating with reduced amplitude' was more frequent in the CC, IDA/ MTD or nEM group. The 'circular gyrating motion' was mainly seen in the CC group $(n=11)$, although in a minority of ciliated edges, but was also present in the nEM group $(n=3)$.

The quantitative parameters are displayed in figure 3. Briefly, the recovery stroke and pause durations were longer in the ODA group. The beating angle, the distance travelled by the cilium tip per beat and the area swept per beat were reduced in the IDA/MTD group (figure 3A). The weighted distance travelled by the cilium tip per second was statistically different between all groups except between the ODA and IDA/MTD groups (figure 3A). When expressed per patient (figure 3B), these results were confirmed, except between the CC and nEM groups for which the difference was not significant.

Except in the 2 DA group, cilia with 'normal motion' (according to our definition given in the Methods) were present in all groups (figure 4A). In the ODA and IDA/MTD groups, only two and three patients, respectively, showed cilia with 'normal motion' (figure 4B). Conversely, in the CC and the nEM groups, 9 and 11 patients, respectively, showed cilia with 'normal motion', despite highly heterogeneous values (figure 4B). Furthermore, in the CC group, no correlation was found between the proportion of cilia with normal ultrastructure and the proportion of cilia with 'normal motion' (data not shown).

Online supplementary videos S5 to S9 show the typical ciliary beating of each ultrastructural phenotypic group.

\section{Ciliary beating and genotype}

Phenotype-genotype correlations were considered for each gene implicated in at least four patients: DNAI1 $(\mathrm{n}=8)$, DNAH5 $(\mathrm{n}=6), \operatorname{CCDC} 39(\mathrm{n}=10)$, RSPH1 $(\mathrm{n}=6)$, RSPH9 $(\mathrm{n}=4)$ and DNAH11 $(\mathrm{n}=13)$.

In the ODA group, the patients with DNAI1 mutations exhibited the most abnormal values regarding the percentage of beating cilia (figure 1), the pause durations, the distance travelled and the area swept (data not shown). Interestingly, the only carrier of a DNAH5 missense mutation, potentially allowing the production of a protein (p.Arg2771Cys), evidenced a remarkably conserved beating: $40 \%$ of beating cilia, normal CBF, normal

Table 2 Qualitative evaluation of ciliary beating: proportion per patient of ciliated edges with each beat pattern (as described by Chilvers et al, ${ }^{13}$ according to the ultrastructural phenotype)

\begin{tabular}{|c|c|c|c|c|c|}
\hline & $2 \mathrm{DA}$ & ODA & IDA/MTD & $\mathrm{CC}$ & $\mathrm{nEM}$ \\
\hline & $n=15$ & $n=15$ & $n=15$ & $n=15$ & $n=15$ \\
\hline Normal beating & $0 \%(0-0)$ & $0 \%(0-59)$ & $0 \%(0-13)$ & $30 \%(0-62)$ & $35 \%(0-50)$ \\
\hline Virtually immotile & $100 \%(100-100)$ & $88 \%(22-92)$ & $64 \%(6-93)$ & $13 \%(0-56)$ & $40 \%(5-85)$ \\
\hline Stiff beating with reduced amplitude & $0 \%(0-0)$ & $10 \%(0-56)$ & $36 \%(7-81)$ & $45 \%(15-63)$ & $30 \%(0-56)$ \\
\hline Circular gyrating motion & $0 \%(0-0)$ & $0 \%(0-0)$ & $0 \%(0-0)$ & $5 \%(0-44)$ & $0 \%(0-15)$ \\
\hline
\end{tabular}

Data are expressed as median (range). Grey boxes point out the specific beat pattern for each ultrastructural phenotype as published by Chilvers et al (nEM group was not evaluated by Chilvers). ${ }^{13}$

CC group, central complex defect; 2 DA group, combined outer/inner dynein arms defect.; IDA/MTD group, inner dynein arm defect with microtubular disorganisation; nEM group, normal ultrastructure; ODA group, outer dynein arm defect. 
A
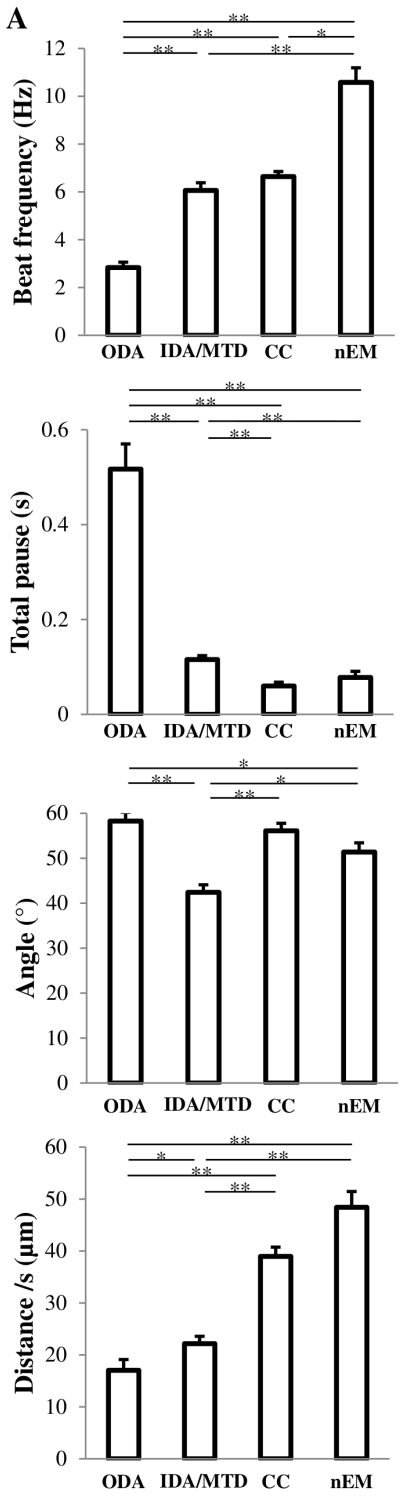
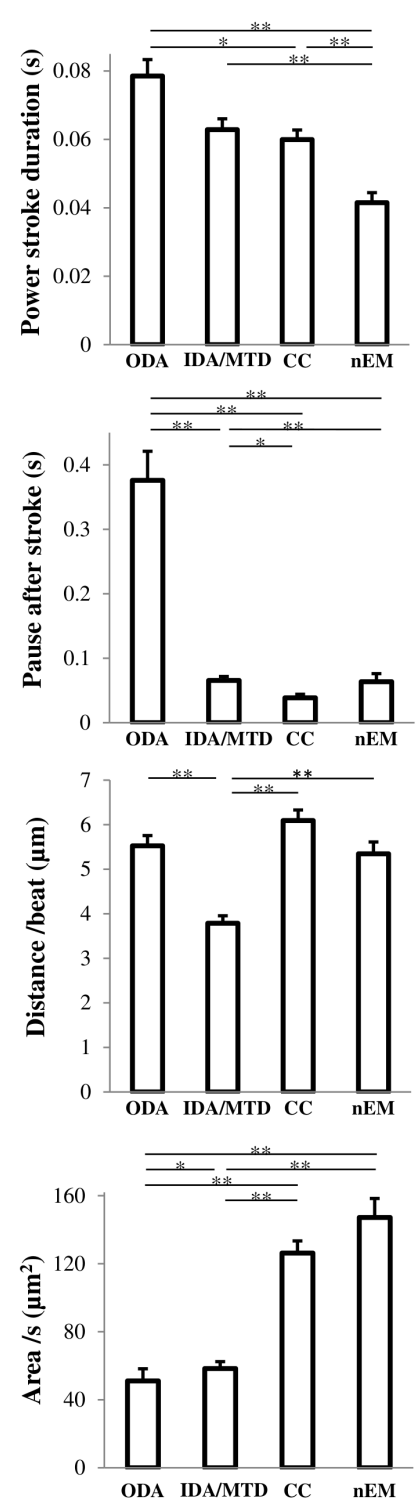
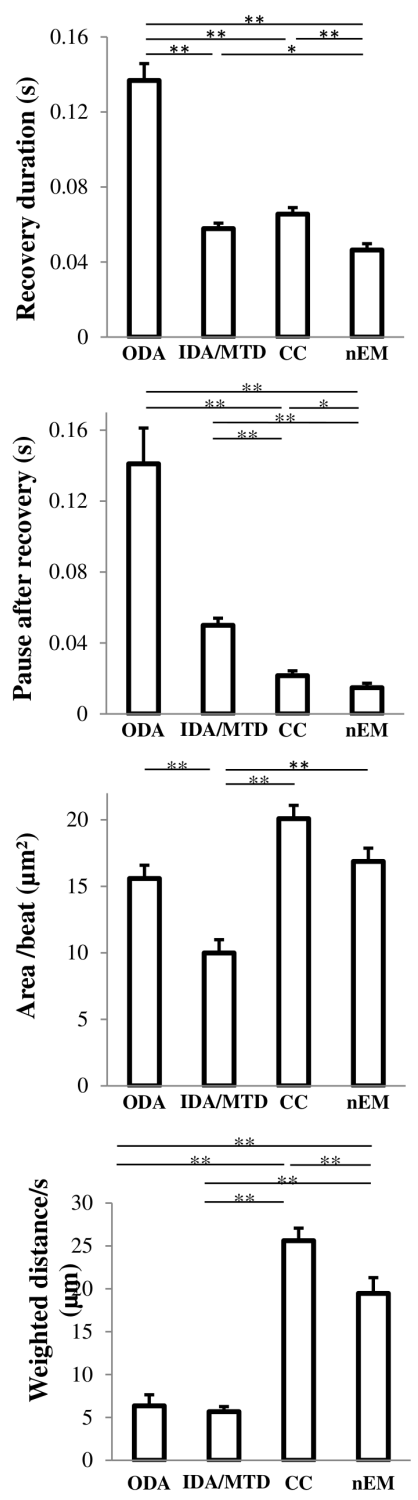

Mutated genes

$\diamond$ DNAH5

$\diamond$ DNAII

- CCDC39

$\square$ CCDC40

- RSPHI

- RSPH9

- DNAJB13

- HYDIN

$\triangle$ DNAHII

$\diamond \square \bigcirc \triangle$ Unknown

Figure 3 Quantitative analysis of ciliary beating. The 2DA group is not represented since all cilia are immotile. (A) Quantitative parameters presented per cilia, as mean and SE. (B) Weighted distance travelled per second by the cilium tip presented per patient. Black bars feature medians. + represents patient with potential production of a full-length protein. ${ }^{*} \mathrm{P}<0.05,{ }^{*} \mathrm{p}<0.001$. Values in disease controls are displayed in online supplementary table $\mathrm{S} 2$. CC group, central complex defect; IDA/MTD group, inner dynein arm defect with microtubular disorganisation; nEM group, normal ultrastructure; ODA group, outer dynein arm defect; 2 DA group, combined outer/inner dynein arms defect.

pause duration, nearly normal weighted distance travelled per second and 50\% of cilia with 'normal motion' (figure 1, 2B, 3B, 4B).

In the IDA/MTD group, the patients with CCDC39 mutations evidenced higher mean CBF than those with CCDC40 mutations (median (range): $6.7 \mathrm{~Hz} \quad(3.8-10.1)$ and $3.3 \mathrm{~Hz} \quad(3.2-5.2)$, respectively, $\mathrm{p}=0.028$ ) (figure $2 \mathrm{~B}$ ). Furthermore, the maximal CBF ranges from 6.1 to $24.2 \mathrm{~Hz}$ (median $13.9 \mathrm{~Hz}$ ) in patients carrying CCDC39 mutations, while the highest CBF was $9.7 \mathrm{~Hz}$ in patients carrying CCDC40 mutations $(p=0.049)$ (figure $2 C$ ). 

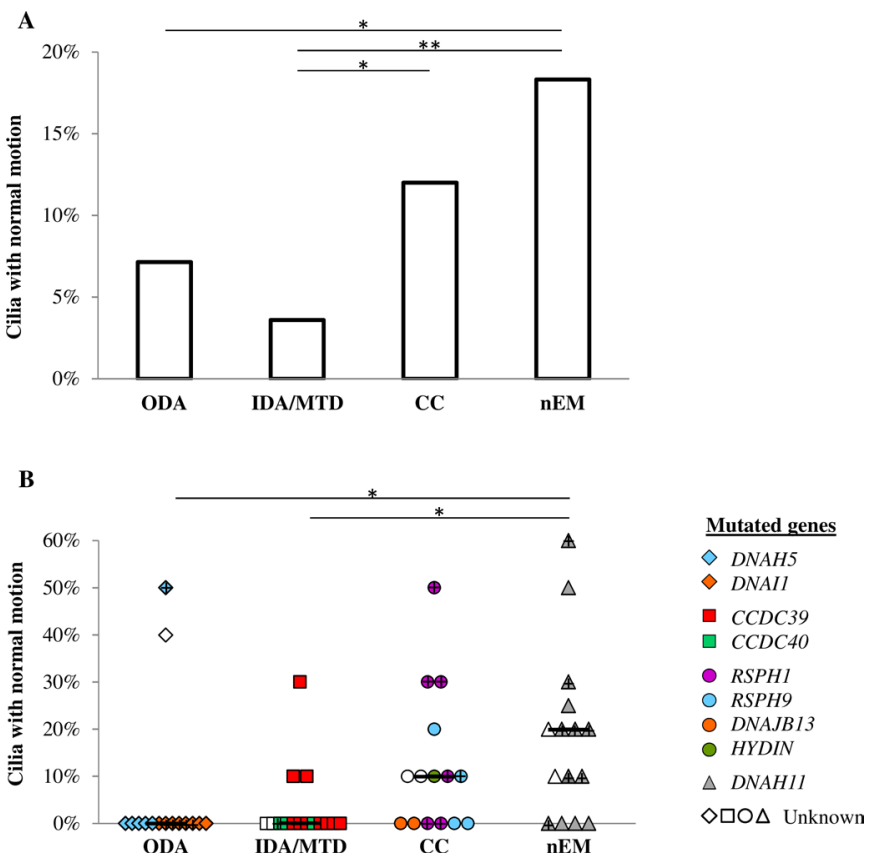

Figure 4 Proportion of beating cilia showing a 'normal motion', defined as a normal beat frequency and a normal angle and a normal distance travelled by the cilium tip per second (as published by Papon et $a^{25}$ ). The 2DA group is not represented since all cilia are immotile. (A) Data are presented per cilia. (B) Data are presented per patient. Black bars feature medians. + represents patient with potential production of a full-length protein. ${ }^{*} \mathrm{P}<0.05,{ }^{*} \mathrm{p}<0.001$. CC group, central complex defect; IDA/MTD group, inner dynein arm defect with microtubular disorganisation; nEM group, normal ultrastructure; ODA group, outer dynein arm defect; 2 DA group, combined outer/inner dynein arms defect.

In the CC group, patients with RSPH1 mutations seem to exhibit less abnormal beating parameters (figures 1, 2B, 3B, $4 \mathrm{~B}$ ), and a less frequent gyrating pattern (RSPH1: 2/6 patients evidenced a gyrating pattern, in up to $10 \%$ of ciliated edges; RSPH9 and DNAJB13: all patients evidenced a gyrating pattern, in up to $40 \%$ of ciliated edges). Interestingly, three patients carrying a mild RSPH1 mutation evidenced at least $30 \%$ of cilia with 'normal motion' (figure 4B) with a normal $\mathrm{nNO}$ for one of them $(194 \mathrm{~nL} / \mathrm{min})$ : (i) as for the c.366G $>$ A (p.Arg122=) silent mutation, located on the first base of exon 5 , although it weakens the intron 4 acceptor splice site, normal RSPH1 transcripts have been detected in that situation (19); (ii) similarly, the c. $727+5 \mathrm{G}>\mathrm{A}$ splice mutation partially impairs the intron 7 donor splice site, probably allowing the production of some normal transcripts; (iii) as for the c.308G >A (p.Gly103Asp) missense mutation, it may be compatible with the production of a protein.

In the nEM group, the patients with DNAH11 mutations showed heterogeneous values, but mostly an increased mean CBF with extremely high maximal CBF (figure 2B, 2C) and occasionally a reduced beating angle or distance travelled per beat (data not shown). The five patients with severe biallelic mutations showed a minority of beating cilia (1\%-32\%) (figure 1) and consequently a systematically impaired weighted distance travelled per second (figure 3B).

\section{DISCUSSION}

This prospective study is the first one that looks for phenotypegenotype correlations in a large cohort of patients with PCD, based on a deep ciliary phenotyping and an extensive genotyping. Considering the numerous limitations of the subjective beat pattern analysis and the $\mathrm{CBF}$, recently highlighted by the American Thoracic Society, we used a quantitative beating analysis by HSV we previously developed. ${ }^{25}$ We found that specific beating parameters differ between the different ultrastructural phenotypes and genotypes. To summarise our main results: a 2DA defect induces a total ciliary immotility, regardless of the gene involved; an ODA defect induces a minimal residual beating with dramatically low CBF related to increased recovery stroke duration and prolonged pauses, especially in case of DNAI1 mutations; an IDA/MTD phenotype induces a low percentage of beating cilia with a decreased beating angle and, in case of CCDC39 mutations, a relatively conserved mean CBF with a high maximal CBF; a CC defect induces nearly normal beating parameters, independently of the involved gene, and a gyrating motion in a minority of ciliated edges, especially in case of RSPH1 mutations and a PCD with normal ultrastructure exhibits heterogeneous values in beating parameters, but frequently an increased CBF with an extremely high maximal CBF related to the cilia high-speed motion.

Although all cilia were found to be immotile when both dynein arms are missing, other studies reported a residual beating in patients with an absence of both dynein arms. ${ }^{13} 1431$ In the latter cases, it would, however, be interesting to know the proportion of abnormal cilia and the expected consequences of the genotype at the protein level.

Regarding the ODA and IDA/MTD groups, the striking feature is the significant decreased weighted distance travelled by the cilium tip. As for the ODA defect, the slow recovery strokes and the very long pauses, which are responsible for the dramatically decreased CBF, are in line with the role of ODA in the initialisation and the force of beating strokes. ${ }^{32} 33$ In cilia with the IDA/ MTD phenotype, the reduced beating angle and distance travelled per beat readily explain the reduced amplitude reported in case of IDA defect, ${ }^{13}$ a result supporting the hypothesis that IDA are 'ODA helpers' increasing the amplitude of the ciliary beating. ${ }^{32}$ Therefore, the slow beating in case of ODA defect and the reduced beating angle in case of IDA/MTD phenotype explain the short distance travelled and the small area swept per second evidenced in these two ultrastructural phenotypes. Given the dramatically low percentage of beating cilia in these two groups, the weighted distance travelled by the cilium tip per second is even more reduced. This is of key importance, as the area swept by the cilium per second is determinant for the quantity of fluid displaced by the beating cilia.

The main characteristic of the CC group is the high even heterogeneous percentage of beating cilia with nearly normal CBF and quantitative beating parameters. However, we evidenced only $2 / 15$ patients with $100 \%$ of beating cilia, when previous studies reported no immotile cilia in case of CC defect. ${ }^{13}$ Reported as a hallmark of CC defect, ${ }^{13}$ we observed a ciliary gyrating motion in only a minority of ciliated edges in the CC group, as previously reported, ${ }^{14}$ and occasionally in case of PCD with normal ultrastructure. Considering the beating pattern was evaluated on ciliated edges mainly viewed from the side, we may have underestimated the proportion of circular gyrating motion but only in case of minimal gyration, as the short focal length of the lens allowed to identify the large majority of beating with a $3 \mathrm{D}$ component. Finally, 8/15 patients with a CC defect displayed a weighted distance travelled by the cilium tip per second above the threshold proposed by Papon to confirm PCD diagnosis. ${ }^{25}$ This highlights the difficulty in diagnosing PCD related to $\mathrm{CC}$ defects, given there is no laterality defect, the clinical 
phenotype could be milder, the nNO values seem less abnormal and the ultrastructural defect is observed in only a minority of cilia. ${ }^{19} 262735$

In PCD with normal ultrastructure, we showed that the qualitative evaluation is not discriminant as all beating patterns are observed, including the gyrating motion, and that the $\mathrm{CBF}$ values are highly variable even within a patient, as already reported. ${ }^{14} 17$ Finally, a high maximal CBF appears to be more characteristic of this phenotype than a high mean CBF. Our quantitative analysis now demonstrates that the increased $\mathrm{CBF}$ is related to the cilia high-speed motion and not to modifications of pause durations or beating angle. It corresponds to the hyperkinetic ciliary beating first reported by Pedersen, ${ }^{12}$ later confirmed in most patients with DNAH11 mutations. ${ }^{14} 16-18$

Our phenotype-genotype correlation study reveals that DNAI1 mutations result in the most abnormal ciliary beating pattern-if we do not consider the total ciliary immotility of patients with 2DA defect. Interestingly, mutations in DNAI1, encoding an intermediate chain localised at the base of the ODA, result in a defect of the whole dynein arm including the heavy chain DNAH5 localised at the distal part, while mutations in DNAH5 could allow the preservation of the basal part of the ODA. ${ }^{36}$ Regarding the patients with DNAH5 mutations, our results are in line with a previous study showing a minimal and uncoordinated ciliary beating with dramatically low CBF. ${ }^{14}$

In patients with CCDC39 mutations, our quantitative analysis shows similar power and recovery strokes durations that could explain the difficulty to distinguish the power and recovery strokes as already reported in patients with CCDC40 mutations. ${ }^{14}$ Noteworthy, our study unveils that the maximal CBF allows discriminating patients with CCDC39 mutations from those with CCDC40 mutations, as CCDC39 mutations result in higher maximal CBF. Despite a similar and interlinked location within the axoneme, the CCDC39 and CCDC40 proteins, which interact with distinct axonemal structures, could differentially drive the ciliary beating. ${ }^{37}$

The patients with RSPH1 or RSPH9 mutations displayed a relatively conserved beating, even if RSPH9 mutations may impair more severely the ciliary beating and may induce more frequently a gyration motion, in line with a previous study reporting a slightly reduced beating in patients with $R S P H 1$ mutations. ${ }^{19}$

Regarding the patients with DNAH11 mutations, besides the well-reported increased CBF, ${ }^{14}{ }^{16-18}$ a decreased amplitude was previously suggested. ${ }^{14}{ }^{16}$ Our results now clearly show a reduced beating angle and distance travelled per beat.

Noteworthy, in the CC and nEM groups, the majority of the patients who have parameters above the median (proportion of beating cilia, mean $\mathrm{CBF}$ and weighted distance) carries a mild mutation consistent with the production of some protein (online supplementary table S3) (figures 1, 2B and 3B). This highlights that the gene and the nature of the mutation may have an effect on the ciliary beating. Finally, HSV seems to be a useful tool for deep phenotyping within a given ultrastructural defect: four patients with at least one mild mutation in either DNAH5 or RSPH1 showed relatively conserved beating parameters while patients with severe DNAH11 mutations showed more an impaired ciliary beating.

\section{CONCLUSION}

Our study proposes a deep ciliary phenotyping, including a quantitative beating analysis by HSV, and an extensive genotyping of a large cohort of patients with PCD. We show that specific beating anomalies are related to a specific ultrastructural phenotype, and consequently to the group of genes implicated in this phenotype.
Furthermore, our data suggest a relationship between some ciliary beating anomalies and specific genes. We also highlight that, for a given causal gene, milder mutations, such as missense variations, could result in milder alterations of the beating parameters. This relation between the implicated gene and/or the severity of the mutation and the ciliary beat impairment suggests subtle genotypephenotype correlations, that need to be investigated with a higher number of patients per genotype.

With the large diffusion of the NGS-based technologies, it is tempting to anticipate that molecular analyses will progressively provide a PCD diagnosis. Thereby, the American Thoracic Society recently recommended using an extended genetic panel as a diagnostic test over TEM, in patients with a high probability of having PCD. ${ }^{9}$ However, considering the high number of PCD genes so far identified ( $>40$ genes) and the size of some of them $(>80$ exons), it is frequent to identify several sequence variants in several PCD genes or to identified variants of unknown significance. In those situations, it is essential to demonstrate a strict concordance between the suspected causal gene and the ciliary phenotype. Our results suggest that HSV including quantitative beating analysis could represent a valuable alternative to TEM in order to guide the molecular analyses towards the best candidate gene(s) to be analysed and/or to assess the implication of identified sequence variants, as recently proposed by Chioccioli to discriminate between DNAH11 or HYDIN variants. ${ }^{15}$ This is of major interest since HSV is easier, cheaper and faster to perform than TEM ( $€ 150$ vs $\sim € 1500$ and $\sim 45$ min vs $\sim 1$ month in our centre). ${ }^{829}$ In summary, genes implicated in 2DA defect would be the best candidates when all cilia are immotile. In case of residual beating with a decreased weighted distance travelled by the cilium tip per second, genes related to ODA defect would be the best candidates if the pause durations are increased and those related to IDA/MTD phenotype if the beating angle is decreased. In case of residual beating with borderline weighted distance travelled by the cilium tip per second, genes related to CC defect would be good candidates if a high percentage of cilia are still beating or if a gyrating motion is identified, while those related to PCD with normal ultrastructure would be selected when cilia are hyperkinetic.

In order to investigate predictive capabilities of HSV and to apply such an algorithm, our data need to be confirmed in a higher number of patients in each phenotype/genotype and if necessary after ciliated cell culture. Such studies on a rare disease must be done through international networks of specialists working on HSV analyses with standardised practice. ${ }^{38}$ Furthermore, these data might be completed in less typical PCD cases, such as partial axonemal defects and for less frequently implicated genes (eg, N-DRC related genes).

\section{Author affiliations}

${ }^{1}$ Universite Paris-Est, Faculte de Médecine, INSERM (Institut National pour la Santé et la Recherche Médicale) UMR_S955, Equipe 13, CNRS (Centre National pour la Recherche Scientifique), ERL 7000, Creteil, France

${ }^{2}$ Department Woman-Mother-Child, Service of Pediatrics, Pediatric Pulmonology Unit, Lausanne University Hospital and University of Lausanne, Lausanne, Switzerland

${ }^{3}$ Sorbonne Universite, Faculte de Medecine, INSERM UMR_S933, APHP (Assistance Publique - Hopitaux de Paris), Unité de génétique moléculaire, Hopital Armand-

Trousseau, Paris, France

${ }^{4}$ APHP, Hopital Armand-Trousseau, Unite de Pneumologie Pédiatrique, Centre National de Reference des Maladies Respiratoires Rares, Creteil, France ${ }^{5}$ Centre Hospitalier Intercommunal de Creteil, Service d'Anatomopathologie, Laboratoire de Microscopie Electronique, Creteil, France

${ }^{6}$ APHP, Groupe Hospitalier Henri Mondor-Albert Chenevier et Centre Hospitalier Intercommunal de Creteil, Service d'ORL et de Chirurgie Cervico-faciale, Creteil, France

7Universite Paris-Sud, Faculte de Medecine, APHP, Hopital Bicetre, Service d'ORL et de Chirurgie Cervico-maxillo-faciale, Le Kremlin Bicetre, France 
Acknowledgements The authors are grateful to the patients and their families who participated in this study and thank all referring physicians for their help and confidence. Electron microscopy studies were performed at the Plateforme d'Imagerie Cellulaire Pitie Salpetriere (PICPS). SB, ML, MB, AT, ACl, ACo, SA, EE, JFP and $\mathrm{BL}$ are members of the RaDiCo-PCD project (ANR-10-COHO-003, INSERM, France) and the EU-funded COST Action BEAT-PCD (Better Evidence to Advance Therapeutic Options for PCD, BM1407).

Contributors Conception and design of the study: SB, ML, EE, JFP, BL. Acquisition, analysis and interpretation of data: $S B, M L, M B, A T, G M, S T, N C, C F, F D, B C, A C l, M F$, $A C O, S A, E E, J F P, B L$. Writing or substantial involvement in revision: $S B, M L, S A, E E$, JFP, BL.

Funding The authors have not declared a specific grant for this research from any funding agency in the public, commercial or not-for-profit sectors.

Competing interests None declared.

Patient consent for publication Not required.

Provenance and peer review Not commissioned; externally peer reviewed.

Data availability statement Data are available on reasonable request.

ORCID iD

Sylvain Blanchon http://orcid.org/0000-0003-0284-9705

\section{REFERENCES}

1 Afzelius BA. A human syndrome caused by immotile cilia. Science 1976;193:317-9.

2 Afzelius BA, Srurgess JM. The immotile-cilia syndrome: a microtubule-associated defect. CRC Crit Rev Biochem 1985;19:63-87.

3 Barbato A, Frischer T, Kuehni CE, Snijders D, Azevedo I, Baktai G, Bartoloni L, Eber E, Escribano A, Haarman E, Hesselmar B, Hogg C, Jorissen M, Lucas J, Nielsen KG, O'Callaghan C, Omran H, Pohunek P, Strippoli M-PF, Bush A. Primary ciliary dyskinesia: a consensus statement on diagnostic and treatment approaches in children. Eur Respir J 2009;34:1264-76.

4 Praveen $\mathrm{K}$, Davis EE, Katsanis N. Unique among ciliopathies: primary ciliary dyskinesia, a motile cilia disorder. F1000Prime Rep 2015;7:36.

5 Kartagener M, Bronchiektasien ZPder. Bronchiektasien bei situs inversus viscerum. Beitr Klin Tuberk 1933:83:489-501.

6 Knowles MR, Daniels LA, Davis SD, Zariwala MA, Leigh MW. Primary ciliary dyskinesia. recent advances in diagnostics, genetics, and characterization of clinical disease. Am J Respir Crit Care Med 2013;188:913-22.

7 Goutaki M, Meier AB, Halbeisen FS, Lucas JS, Dell SD, Maurer E, Casaulta C, Jurca M, Spycher BD, Kuehni CE. Clinical manifestations in primary ciliary dyskinesia: systematic review and meta-analysis. Eur Respir J 2016;48:1081-95.

8 Lucas JS, Barbato A, Collins SA, Goutaki M, Behan L, Caudri D, Dell S, Eber E, Escudier E, Hirst RA, Hogg C, Jorissen M, Latzin P, Legendre M, Leigh MW, Midulla F, Nielsen KG, Omran H, Papon J-F, Pohunek P, Redfern B, Rigau D, Rindlisbacher B, Santamaria F, Shoemark A, Snijders D, Tonia T, Titieni A, Walker WT, Werner C, Bush A, Kuehni CE. European respiratory Society guidelines for the diagnosis of primary ciliary dyskinesia. Eur Respir J 2017:49.

9 Shapiro AJ, Davis SD, Polineni D, Manion M, Rosenfeld M, Dell SD, Chilvers MA, Ferkol TW, Zariwala MA, Sagel SD, Josephson M, Morgan L, Yilmaz O, Olivier KN, Milla C, Pittman JE, Daniels MLA, Jones MH, Janahi IA, Ware SM, Daniel SJ, Cooper ML, Nogee LM, Anton B, Eastvold T, Ehrne L, Guadagno E, Knowles MR, Leigh MW, Lavergne $V$, American Thoracic Society Assembly on Pediatrics. Diagnosis of primary ciliary dyskinesia. An official American thoracic Society clinical practice guideline. Am I Respir Crit Care Med 2018:197:e24-39.

10 Lavie M, Amirav I. In defense of high-speed video microscopy in evaluating patients with suspected primary ciliary dyskinesia. Am J Respir Crit Care Med.

11 Rossman CM, Forrest JB, Lee RM, Newhouse AF, Newhouse MT. The dyskinetic cilia syndrome; abnormal ciliary motility in association with abnormal ciliary ultrastructure. Chest 1981:80:860-5.

12 Pedersen M. Specific types of abnormal ciliary motility in Kartagener's syndrome and analogous respiratory disorders. A quantified microphoto-oscillographic investigation of 27 patients. Eur J Respir Dis Supp/ 1983;127:78-90.

13 Chilvers MA, Rutman A, O'Callaghan C. Ciliary beat pattern is associated with specific ultrastructural defects in primary ciliary dyskinesia. J Allergy Clin Immunol 2003:112:518-24

14 Raidt J, Wallmeier J, Hjeij R, Onnebrink JG, Pennekamp P, Loges NT, Olbrich H, Häffner $\mathrm{K}$, Dougherty GW, Omran $\mathrm{H}$, Werner $\mathrm{C}$. Ciliary beat pattern and frequency in genetic variants of primary ciliary dyskinesia. Eur Respir J 2014:44:1579-88.

15 Chioccioli M, Feriani L, Nguyen Q, Kotar J, Dell SD, Mennella V, Amirav I, Cicuta P. Quantitative High-Speed Video Profiling Discriminates between DNAH11 and HYDIN Variants of Primary Ciliary Dyskinesia. Am J Respir Crit Care Med 2019;199:1436-8.

16 Schwabe GC, Hoffmann K, Loges NT, Birker D, Rossier C, de Santi MM, Olbrich H, Fliegauf M, Failly M, Liebers U, Collura M, Gaedicke G, Mundlos S, Wahn U, Blouin
J-L, Niggemann B, Omran H, Antonarakis SE, Bartoloni L. Primary ciliary dyskinesia associated with normal axoneme ultrastructure is caused by DNAH11 mutations. Hum Mutat 2008;29:289-98

17 Pifferi M, Michelucci A, Conidi ME, Cangiotti AM, Simi P, Macchia P, Boner AL. New DNAH11 mutations in primary ciliary dyskinesia with normal axonemal ultrastructure. Eur Respir J 2010;35:1413-6.

18 Knowles MR, Leigh MW, Carson JL, Davis SD, Dell SD, Ferkol TW, Olivier KN, Sage SD, Rosenfeld M, Burns KA, Minnix SL, Armstrong MC, Lori A, Hazucha MJ, Loges NT, Olbrich H, Becker-Heck A, Schmidts M, Werner C, Omran H, Zariwala MA, Genetic Disorders of Mucociliary Clearance Consortium. Mutations of DNAH11 in patients with primary ciliary dyskinesia with normal ciliary ultrastructure. Thorax 2012;67:433-41.

19 Knowles MR, Ostrowski LE, Leigh MW, Sears PR, Davis SD, Wolf WE, Hazucha MJ, Carson JL, Olivier KN, Sagel SD, Rosenfeld M, Ferkol TW, Dell SD, Milla CE, Randell SH, Yin W, Sannuti A, Metjian HM, Noone PG, Noone PJ, Olson CA, Patrone MV, Dang H, Lee H-S, Hurd TW, Gee HY, Otto EA, Halbritter J, Kohl S, Kircher M, Krischer J, Bamshad MJ, Nickerson DA, Hildebrandt F, Shendure J, Zariwala MA. Mutations in RSPH1 Cause Primary Ciliary Dyskinesia with a Unique Clinical and Ciliary Phenotype. Am J Respir Crit Care Med 2014:189:707-17.

20 Dresdner RD, Wong LB. Measurement of ciliary beat frequency using high-speed video microscopy. Biomed Sci Instrum 1984;20:37-46.

21 Chilvers MA, O'Callaghan C. Analysis of ciliary beat pattern and beat frequency using digital high speed imaging: comparison with the photomultiplier and photodiode methods. Thorax 2000:55:314-7.

22 Rubbo B, Shoemark A, Jackson CL, Hirst R, Thompson J, Hayes J, Frost E, Copeland F, Hogg C, O'Callaghan C, Reading I, Lucas IS, National PCD Service, UK. Accuracy of High-Speed Video Analysis to Diagnose Primary Ciliary Dyskinesia. Chest 2019:155:1008-17.

23 Jackson CL, Behan L, Collins SA, Goggin PM, Adam EC, Coles JL, Evans HJ, Harris A, Lackie P, Packham S, Page A, Thompson J, Walker WT, Kuehni C, Lucas JS. Accuracy of diagnostic testing in primary ciliary dyskinesia. Eur Respir J 2016;47:837-48.

24 Kempeneers C, Seaton C, Chilvers MA. Variation of ciliary beat pattern in three different beating planes in healthy subjects. Chest 2017;151:993-1001.

25 Papon J-F, Bassinet L, Cariou-Patron G, Zerah-Lancner F, Vojtek A-M, Blanchon S, Crestani B, Amselem S, Coste A, Housset B, Escudier E, Louis B. Quantitative analysis of ciliary beating in primary ciliary dyskinesia: a pilot study. Orphanet J Rare Dis 2012:7:78.

26 Vallet C, Escudier E, Roudot-Thoraval F, Blanchon S, Fauroux B, Beydon N, Boulé M, Vojtek AM, Amselem S, Clément A, Tamalet A. Primary ciliary dyskinesia presentation in 60 children according to ciliary ultrastructure. Eur J Pediatr 2013;172:1053-60.

27 Davis SD, Ferkol TW, Rosenfeld M, Lee H-S, Dell SD, Sagel SD, Milla C, Zariwala MA, Pittman JE, Shapiro AJ, Carson JL, Krischer JP, Hazucha MJ, Cooper ML, Knowles MR, Leigh MW. Clinical features of childhood primary ciliary dyskinesia by genotype and ultrastructural phenotype. Am J Respir Crit Care Med 2015;191:316-24.

28 Papon JF, Coste A, Roudot-Thoraval F, Boucherat M, Roger G, Tamalet A, Vojtek AM, Amselem S, Escudier E. A 20-year experience of electron microscopy in the diagnosis of primary ciliary dyskinesia. Eur Respir J 2010;35:1057-63.

29 Shoemark A, Dixon M, Corrin B, Dewar A. Twenty-Year review of quantitative transmission electron microscopy for the diagnosis of primary ciliary dyskinesia. J Clin Pathol 2012:65:267-71.

30 Richards S, Aziz N, Bale S, Bick D, Das S, Gastier-Foster J, Grody WW, Hegde M, Lyon E, Spector E, Voelkerding K, Rehm HL,ACMG Laboratory Quality Assurance Committee. Standards and guidelines for the interpretation of sequence variants: a joint consensus recommendation of the American College of medical genetics and genomics and the association for molecular pathology. Genet Med 2015;17:405-23.

31 Stannard WA, Chilvers MA, Rutman AR, Williams CD, O'Callaghan C. Diagnostic testing of patients suspected of primary ciliary dyskinesia. Am I Respir Crit Care Med 2010;181:307-14.

32 Sturgess JM, Turner JA. Ultrastructural pathology of cilia in the immotile cilia syndrome. Perspect Pediatr Pathol 1984;8:133-61.

33 Wanner A, Salathé M, O'Riordan TG. Mucociliary clearance in the airways. Am J Respir Crit Care Med 1996;154:1868-902.

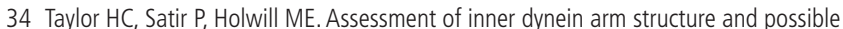
function in ciliary and flagellar axonemes. Cell Motil Cytoskeleton 1999;43:167-77.

35 Kott E, Legendre M, Copin B, Papon J-F, Dastot-Le Moal F, Montantin G, Duquesnoy P, Piterboth W, Amram D, Bassinet L, Beucher J, Beydon N, Deneuville E, Houdouin V Journel H, Just J, Nathan N, Tamalet A, Collot N, Jeanson L, Le Gouez M, Vallette B, Vojtek A-M, Epaud R, Coste A, Clement A, Housset B, Louis B, Escudier E, Amselem S. Loss-Of-Function mutations in RSPH1 cause primary ciliary dyskinesia with centralcomplex and radial-spoke defects. Am J Hum Genet 2013;93:561-70.

36 Fliegauf M, Olbrich H, Horvath J, Wildhaber JH, Zariwala MA, Kennedy M, Knowles MR, Omran H. Mislocalization of DNAH5 and DNAH9 in respiratory cells from patients with primary ciliary dyskinesia. Am J Respir Crit Care Med 2005;171:1343-9.

37 Oda T, Yanagisawa H, Kamiya R, Kikkawa M. A molecular ruler determines the repeat length in eukaryotic cilia and flagella. Science 2014;346:857-60. 